\title{
Revisiting and revising the purinosome
}

Cite this: Mol. BioSyst., 2014, 10, 369

Received 11th September 2013, Accepted 11th December 2013

DOI: $10.1039 / \mathrm{c} 3 \mathrm{mb} 70397 \mathrm{e}$

www.rsc.org/molecularbiosystems

\author{
Alice Zhao, ${ }^{a}$ Mark Tsechansky, ${ }^{b}$ Andrew D. Ellington ${ }^{a}$ and Edward M. Marcotte ${ }^{{ }^{a}}$
}

Some metabolic pathway enzymes are known to organize into multi-enzyme complexes for reasons of catalytic efficiency, metabolite channeling, and other advantages of compartmentalization. It has long been an appealing prospect that de novo purine biosynthesis enzymes form such a complex, termed the "purinosome." Early work characterizing these enzymes garnered scarce but encouraging evidence for its existence. Recent investigations led to the discovery in human cell lines of purinosome bodies-cytoplasmic puncta containing transfected purine biosynthesis enzymes, which were argued to correspond to purinosomes. New discoveries challenge both the functional and physiological relevance of these bodies in favor of protein aggregation.

\section{Introduction}

Multi-enzyme complexes often engage in various forms of substrate channeling, in which sequential pathway enzymes "hand off" intermediate metabolic products amongst each other rather than release them into bulk solution. The advantages of such complexes include improved efficiency and optimized usage of short-lived intermediates. ${ }^{1-5}$

One such possible complex involves the enzymes for de novo purine biosynthesis. Termed the "purinosome," the complex's existence has long been suggested by circumstantial evidence. The search for direct in vivo evidence of the purinosome culminated in the discovery of intracellular punctate bodies formed in part by purine biosynthesis enzymes. ${ }^{6}$ It was argued, based in part upon evidence for purine dependency, that these were functional complexes. ${ }^{6}$ However, new discoveries support a model where these bodies behave in a manner unlike that expected for functional purinosomes and instead have numerous features one might expect of simple protein aggregates or stress bodies. ${ }^{7}$ Here we review the literature evidence characterizing purinosome bodies, and discuss the models that involve metabolically active associations as opposed to general protein aggregation.

\section{Purine biosynthesis}

Purines are ubiquitous and essential components of DNA and RNA, and their derivatives participate in numerous biological processes. Adenine and guanine nucleotides are derived from the compound inosine monophosphate (IMP), which is synthesized

\footnotetext{
${ }^{a}$ Center for Systems and Synthetic Biology, Institute for Cellular and Molecular Biology, University of Texas at Austin, Austin, TX 78712, USA.

E-mail: marcotte@icmb.utexas.edu

${ }^{b}$ Department of Chemistry, Cambridge University, Lensfield Road, Cambridge, UK
}

de novo from phosphoribosyl pyrophosphate (PRPP) through a highly conserved multi-step de novo purine biosynthesis pathway. In higher eukaryotes (such as humans), the pathway consists of six enzymes catalyzing ten sequential reactions converting PRPP to IMP (Table 1). De novo purine biosynthesis activity is up-regulated when the cellular demand for purines exceeds that supplied by the purine salvage pathway, a single-step conversion of hypoxanthine to IMP catalyzed by hypoxanthine phosphoribosyltransferase (HPRT). Conversely, de novo biosynthesis is down-regulated when exogenous purine, i.e. hypoxanthine, is available.

\section{The search for a complex}

The hypothesis that the de novo purine biosynthesis enzymes organize into a multi-enzyme complex has long been attractive based at least in part on the chemical instability of 5-phosphoribosylamine, the first intermediate substrate in the pathway, which suggests an essential direct transfer between PPAT and GART. ${ }^{8}$ Additionally, the consolidation of several individual enzymatic functions onto single bifunctional or trifunctional polypeptide chains has been observed in many organisms, ${ }^{9,10}$ which suggests that stable physical interactions between these enzymes may exist even in organisms which do not consolidate these enzymes on a single polypeptide chain. ${ }^{11}$ The joining of the non-sequential steps 2,3 , and 5 into a single trifunctional enzyme in humans also suggests that this polypeptide may be further, non-covalently juxtaposed with the enzyme for step 4 .

However, historic experiments to isolate an intact purinosome have been largely unsuccessful. Kinetic studies revealed evidence for substrate channeling between PPAT and GART, but attempts to detect physical protein-protein interactions failed. ${ }^{12}$ Later studies found that pairs of purine biosynthesis pathway members 
Table 1 Six human enzymes catalyze the ten-step conversion of phosphoribosyl pyrophosphate to inosine monophosphate

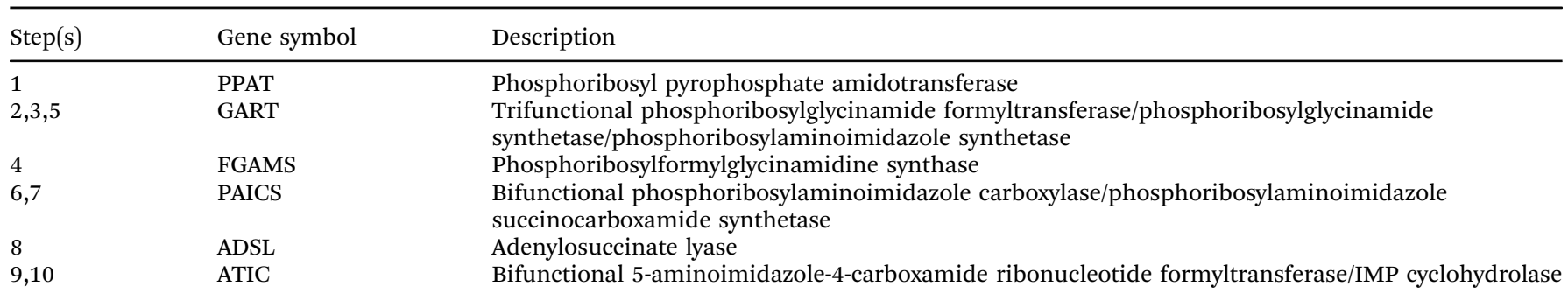

(including PPAT and GART, and ATIC and GART) could be enriched by co-fractionation under some conditions, ${ }^{13-15}$ and purine-dependent protein-protein interactions have been observed using a luciferase reporter system. ${ }^{16}$ Even so, transfected recombinant GART was not found to be co-localized to any cellular architecture that might serve as a structural scaffold to assemble a multi-enzyme complex, ${ }^{17}$ and no biophysical support was found for a larger multi-enzyme complex or a fully intact purinosome. ${ }^{13-15}$ While a complex representing the purinosome may physically exist, it may form only transiently or in a condition-specific manner.

\section{The discovery of purinosome bodies}

In 2008, the human purinosome was thought to have finally been identified by the discovery that fluorescent protein-tagged recombinant purine biosynthesis enzymes form intracellular punctate bodies when transiently expressed in human cell culture. ${ }^{6}$ Bodies formed by FGAMS-GFP were shown to co-localize with bodies formed by the five other enzymes of the pathway, suggesting that the bodies contained all direct participants of the de novo purine biosynthesis pathway. These so-called "purinosome bodies" apparently formed in purine-depleted medium and disassembled in purine-rich medium, a potentially strong indication of function. Moreover, microtubule inhibitors that disfavored purinosome body formation also led to decreased purine synthesis. ${ }^{18}$ Finally, it has been shown that purinosome body assembly is under casein kinase II (CK2) and GPCR control, and could be modulated by heat shock chaperones, ${ }^{7,19-21}$ and in parallel, modulation of CK2 and GPCR activities has been shown to correlate with changes in the overall spatial distribution of bulk intracellular protein. ${ }^{20}$

\section{Conflicting evidence}

While there is supporting evidence for the hypothesis that purinosome bodies form in a purine-dependent and reversible manner, it is possible that these effects are not solely due to the presence or absence of purines. For example, while it has been reported that purinosome bodies form when cells are cultured in purine-depleted medium, the specific growth medium employed for these experiments actually significantly differed from the purine-rich control medium in ways that went beyond the mere presence or absence of purines. The purine-depleted medium was generated by serum dialysis with a 25000 Da pore size, which would have removed a variety of compounds other than purines (which are only 100-500 Da); additionally, the serum supplementation was doubled for the "purine-rich" medium. ${ }^{6}$ These non-conservative changes confound interpretation of the purinedependency of the observations. Notably, in other experiments, altering only purine levels did not affect purinosome body formation: purinosome bodies were unaffected after specifically adding an exogenous purine source (hypoxanthine) back to "purine-depleted" medium, ${ }^{6}$ or after specifically adding a purine antagonist (azaserine). ${ }^{6}$ These contrary observations suggest that the bodies may not form in response to purine levels, but are instead dependent on other factors (we will suggest aggregation, below). To further complicate matters, independent laboratories have reported both induction and no induction of purinosome body formation by similar bulk nutrient-depletion experiments, ${ }^{6,7,22,23}$ further suggesting that unknown factors unrelated to purine concentration might account for the observed phenomena.

As structures for enhancing purine synthesis, purinosomes should exhibit abundant metabolic flux. It has been shown that flux through the de novo purine biosynthesis pathway is suppressed when purinosome body assembly is disrupted by nocodazole. While this correlation supports a role for microtubules in establishing purinosome bodies in live cells ${ }^{18}$ these observations may also arise from an alternative explanation, namely that given that nocodazole is a cell cycle arresting agent, the combination of nutrient-poor medium and arrested cell cycle might also be expected to greatly impede metabolic flux. Such experiments illustrate the intrinsic difficulties in distinguishing flux contributed by the bodies and flux contributed by the free, un-localized pool of the same enzymes. Experiments have so far monitored ${ }^{14} \mathrm{C}$-glycine incorporation into the pool of cellular purines and measured the complete cellular complement of purine biosynthetic enzymes, not explicitly flux through the bodies; to date, no experiments have demonstrated that the bodies themselves provide any metabolic flux. Thus, further kinetic experiments are required before purinosome bodies meet the standard of proof associated with other well-characterized metabolic enhancing structures like acetyl-CoA carboxylase polymers $^{24-27}$ or quaternary structures verified for substrate channeling, such as for tryptophan synthase. ${ }^{4,28,29}$

Additionally, with one exception, ${ }^{22}$ all literature evidence for purinosome bodies has thus far relied on transiently expressed recombinant fluorescent protein fusion constructs. This may be a consequence of reported difficulties and possible artifacts surrounding native immunofluorescent labeling of purine biosynthesis enzymes. ${ }^{6,7}$ For example, it was observed that while 
endogenous GART behaved similarly to the GART fusion construct in "purine-depleted" medium, its endogenous behavior did not correspond to that observed for its fusion construct in "purinerich" medium. ${ }^{6}$ The sole reliance on microscopy with fusion proteins, and the absence of other physical demonstrations of the purinosome as a whole, argues for caution in interpretation.

\section{What are purinosome bodies?}

Somewhat problematically, published purinosome bodies have varied widely in their morphologies, ranging from pinpoint foci to oil droplet-like, and methods have not been developed yet either to classify purinosome bodies or to distinguish them from aggregates based on morphology. In our own studies, we have observed a dynamic spectrum of morphologies, as well as both increases and decreases in the numbers of bodies per cell even over the course of unperturbed growth. ${ }^{7}$ Future work clearly remains to address and characterize the different morphologies and kinetics of purinosome bodies, and to discover cellular markers that can securely distinguish bona fide purinosomes, should they exist. ${ }^{30}$

Another issue that confounds interpretation is that it is unclear whether the purine enzyme fusion constructs function similarly to endogenous enzymes. Others have observed that fluorescent protein fusions are subject to aggregation. ${ }^{31,32}$ Even if we assume that some fluorescent protein fusions function similarly to the native proteins, there is large heterogeneity observed in purinosome body formation. The penetrance of body formation following transient transfection varies widely across enzymes, with individual enzyme body formation rates ranging from $5 \%$ to $77 \%$ of the transfected cells. ${ }^{6,7}$ This heterogeneity, persisting even in cell populations treated with pharmacophores that promote purinosome bodies (15 to $95 \%$ penetrance of assembly), ${ }^{19}$ is inconsistent with the hypothesis that each body contains all members of the pathway, and at a minimum, such bodies are unlikely to represent complete purinosomes.

The constituents of purinosome bodies as measured by microscopy are also sometimes in disagreement with biochemicallycaptured purine enzyme protein-protein associations. As previously shown through partial co-purification, two folate metabolism enzymes (serine hydroxymethyltransferase (SHMT1) and C1THF synthase) associate with the folate-utilizing purine biosynthesis enzymes GART and ATIC. ${ }^{15,33}$ GART's catalytic activity actually requires interaction with $\mathrm{C} 1 \mathrm{THF}$ synthase or its analog. ${ }^{33,34}$ However, these two folate metabolism enzymes were found to be excluded from purinosome bodies. ${ }^{6,21}$ Meanwhile, an additional folate enzyme MTHFS (which catalyzes the conversion of a product from SHMT1 into substrate for C1THF synthase) was independently discovered to co-localize to purinosome bodies. ${ }^{23}$ Beyond C1THF synthase, a multitude of additional proteins not previously implicated in purine biosynthesis have been localized to purinosome bodies, including Hsp70, Hsp90, ubiquitin, Bag2, Bag5, Stip1/Hop, p23, DnaJ-C7 (Hsp40), and DnaJ-A1 (Hsp40). ${ }^{7,21}$ Many of these proteins found associated with the purinosome body are commonly associated with deleterious protein aggregates. ${ }^{35-44}$ The relatively high rate of co-localization between an individual purine biosynthesis enzyme and Hsp70 compared to that between pairs of purine biosynthesis enzymes gives rise to the hypothesis that the purinosome bodies are non-specific aggregates rather than specific functional metabolic complexes. ${ }^{7}$

\section{Growing evidence for protein aggregation}

Many enzymes can aggregate under conditions of cellular stress or recombinatorial expression, and as we discussed above, the medium used to observe inducibility of purinosome bodies was broadly depleted of nutrients. ${ }^{6}$ Unsurprisingly, a switch to this nutrient-depleted medium might be stressful for some cells (e.g., HTB-125 cells cannot survive in the depleted medium). ${ }^{6}$ It is therefore possible that this stress contributed to the visible aggregation of recombinant purine enzyme constructs as stress bodies, which would then have been reversed by providing the cell with nutrients necessary to dissociate or clear aggregated protein bodies. Our study in 2013 supports this simpler explanation that purinosome bodies may be aggregated proteins that commonly result from fusion protein expression. ${ }^{7}$ Many characteristics of purinosome bodies were found to be shared between those of canonical protein aggregates, including body morphology, inducibility by diverse stress, co-localization with ubiquitin and chaperones, and correlation with transfected DNA quantity, protein aggregation potential, and increased cell death. ${ }^{7}$ In this regard, purinosome bodies exhibit overlapping co-localization with a recombinant fusion protein shown to mark aggregates and aggresomes $^{21,31}$ (Fig. 1), and many literature figures of purinosome bodies closely resemble the spectrum of morphologies formed by disease-associated protein aggregates, including both huntingtin and $\alpha$-synuclein (Fig. 2). In accordance with the notion that
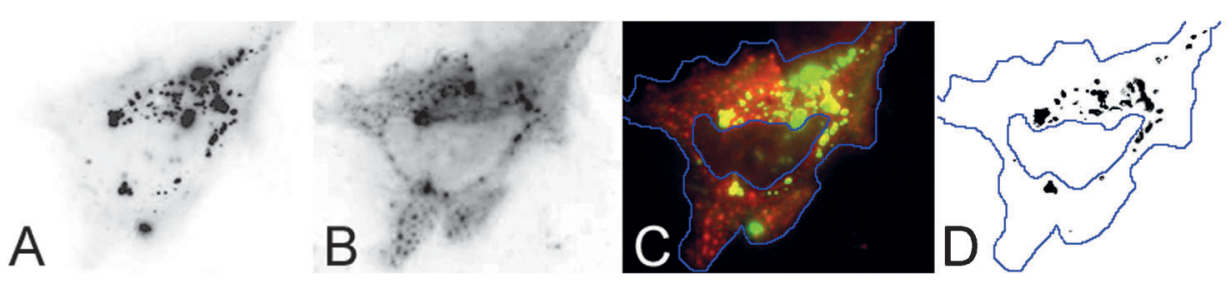

Fig. 1 Partial co-localization of [A] gp-250 (also known as GFP-250) with [B] FGAMS-OFP, co-transfected in the same cell, as reported by French et al. ${ }^{21}$ The overlay in $[C]$ shows the merge of panel (A) in green and panel (B) in red (Pearson's coefficient of 0.4 and overlap coefficient of 0.41 ), ${ }^{21}$ with regions of co-localized expression extracted and plotted in [D] for clarity. Notably, over-expression of the GFP-chimera, gp-250, has been reported to form insoluble aggregates that are delivered to aggresomes. ${ }^{31,63}$ Reprinted with permission from the Proceedings of the National Academy of Sciences. 

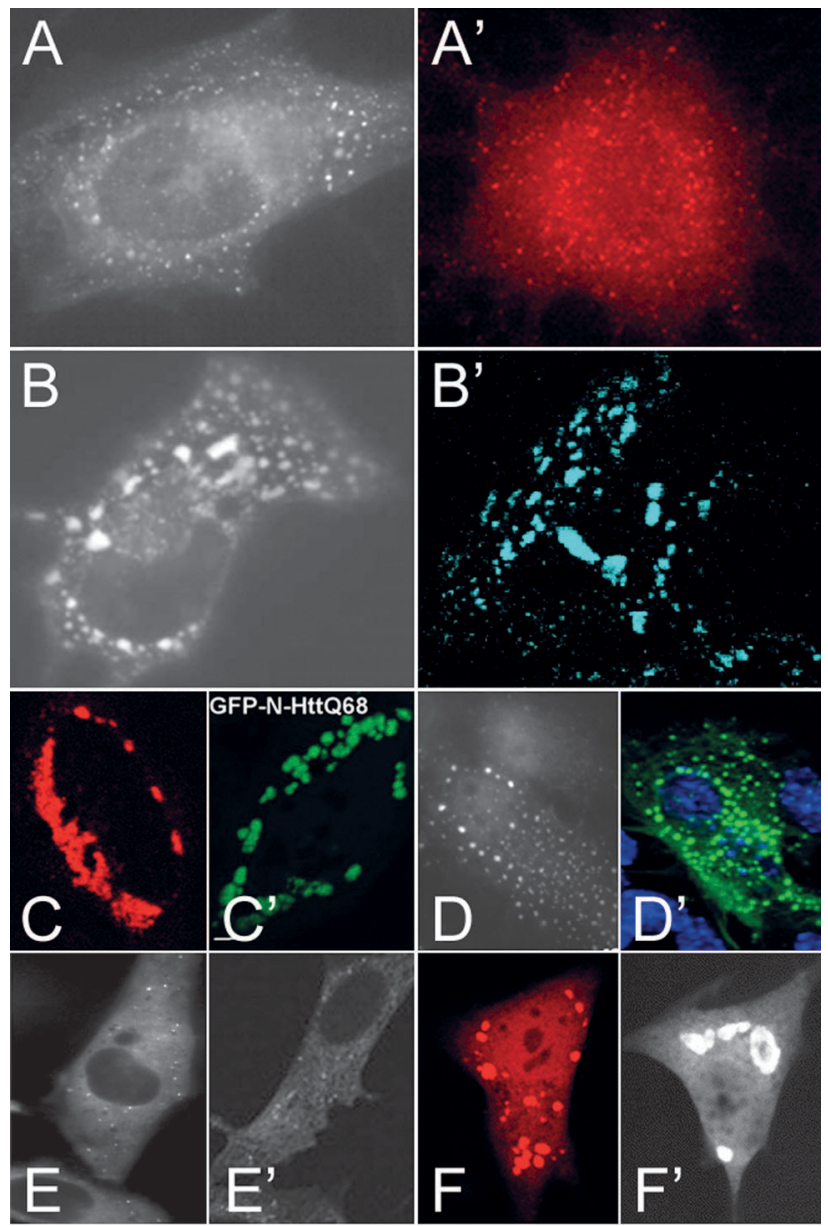

Fig. 2 Intracellular purinosome bodies display a spectrum of morphologies similar to bodies formed by canonical protein aggregates. $[\mathrm{A}-\mathrm{F}]$ are purinosome bodies formed in HeLa cells. $\left[\mathrm{A}^{\prime}-\mathrm{F}^{\prime}\right]$ are various aggregated disease-associated protein inclusions formed in various mammalian cell lines. We can roughly classify these representative (but not exhaustive) morphologies into arbitrary categories: pinpoint foci include [A] TrifGARTGFP from An et al. $2008^{6}$ and $\left[A^{\prime}\right] \alpha$-synuclein aggregates in oligodendroglial cells, from Riedel et al. ${ }^{66}$ Droplet-like clusters are evident in [B] FGAMS-GFP from An et al. $2008^{6}$ and $\left[\mathrm{B}^{\prime}\right]$ a mutated form of glial fibrillary acidic protein (GFAP) attached to CFP transiently transfected into modified astrocytes, from Mignot et al. ${ }^{67}$ Bulk clumps are shown in [C] FGAMS-OFP transiently expressed in HeLa cells from Field et al. ${ }^{23}$ and [ $\left.\mathrm{C}^{\prime}\right]$ GFP-tagged huntingtin fragment with polyglutamine expansion transiently expressed in HeLa cells, from Bjørkøy et al. ${ }^{68}$ Spherical puncta are shown in [D] FGAMS-OFP from An et al. JBC $2010^{19}$ and [D'] GFAP-GFP transiently transfected into astrocytes, from Mignot et al. ${ }^{67}$ Sparse pinpoint foci are formed by [E] FGAMS-GFP from An et al. PNAS 2010, ${ }^{18}$ and $\left[E^{\prime}\right]$ a fragment of p62 protein, a common component of disease-associated protein aggregates, ${ }^{69-72}$ tagged to GFP and transiently expressed in NIH3T3 fibroblasts (although the authors Bjørkøy et al. ${ }^{68}$ classify this subtler morphology as diffuse). Large oildroplet-like bodies are formed by [F] hTrifGART-OFP transfected in HeLa cells from Field et al. $^{23}$ and $\left[\mathrm{F}^{\prime}\right] \alpha$-synuclein-EGFP transfected into $\mathrm{H} 4$ neuroglioma cells, from McLean et al. ${ }^{73}$ All images are reproduced with permission from the respective publishers.

aggregated proteins are inherently cytotoxic, ${ }^{45}$ cells exhibiting purinosome bodies were also found to be associated with early cell death, ${ }^{7}$ although it is unclear whether the bodies were a cause of that stress or rather an indicator of stressed cells.
Follow-up studies characterizing purinosome bodies as functional assemblies have also uncovered a surprising number of additional features that parallel features of disease-associated aggregates. For example, as pointed out earlier, inhibition of microtubule polymerization with nocodazole blocks formation of purinosome bodies and reduces cellular flux of de novo purine biosynthesis. ${ }^{18}$ Likewise, nocodazole also blocks formation of inclusion bodies and aggresomes (bodies containing protein aggregates $)^{46-48}$ and places cells in $\mathrm{G} 2 / \mathrm{M}$ arrest. In a similar fashion, partial inhibition of casein kinase 2 (CK2) by small molecule inhibitors was found to induce purinosome body formation. ${ }^{19}$ Inhibition of CK2 is also known to disrupt hundreds of cellular processes, ${ }^{49}$ among them being protein homeostasis which regulates protein aggregation..$^{50,51}$ Notably, CK2 inhibitors are highly non-specific, ${ }^{52}$ while some induce oxidative stress ${ }^{53,54}$ (a promoter of purinosome body formation) ${ }^{7}$ and apoptosis. ${ }^{55}$ Additionally, the observation that CK2 inhibitors affect FGAMS, GART, and PPAT but do not affect PAICS, ADSL, or ATIC unless a member from the latter set is co-transfected with one in the former ${ }^{19}$ suggests either that individual members of the pathway are differentially and complexly regulated to assemble bodies or that these observations may be transfection artifacts. As well, the claim that the formation of purinosome bodies can be controlled by the addition of $\mathrm{G}_{\alpha \mathrm{i}}$ agonists or antagonists ${ }^{20}$ may again be more simply explained by aggregation: downstream $\mathrm{G}_{\alpha \mathrm{i}}$ targets (e.g., the PI3K/Akt pathway) include regulators of various stress-related cellular responses, such as cell survival and protein synthesis, which will also influence protein homeostasis and aggregation. Notably, G protein signaling has been implicated in regulating autophagy (a mechanism known to clear aggregated proteins $)^{56,57}$ through $\mathrm{G}_{\alpha \mathrm{i}}$-control of autophagic sequestration ${ }^{58,59}$ and through autophagic vacuole formation. ${ }^{60}$

While it appears that purinosome bodies may be the result of stress-induced aggregation, it is still not entirely clear what they are. Purinosome bodies are distinct from stress granules ${ }^{21}$ (messenger ribonucleoprotein bodies composed of translation initiation factors and mRNAs), ${ }^{61,62}$ but additional work is necessary to differentiate them from other forms of stress-inducible bodies (Table 2). While some data suggest that chaperones promote purinosome body assembly, observations supporting the contrary also exist. Experiments perturbing the activity of heat shock chaperones (e.g., Hsp70 or Hsp90) by pharmacophores can promote purinosome body assembly or disassembly depending on the drug concentration and treatment duration, ${ }^{7,21}$ and further study is necessary to clarify the disparity.

\section{Speculations and conclusions}

The idea of a purinosome has many compelling features. Assorted evidence ranging from metabolic flux and channeling considerations to pairwise kinetic or physical interactions observed between particular biosynthetic enzymes supports the idea of some form of physical association between purine biosynthetic enzymes. Thus, there is a substantial body of literature suggesting the existence of the purinosome. Here, we address confounding issues surrounding 
Table 2 Some of the protein-rich bodies identified in the eukaryote cytosol

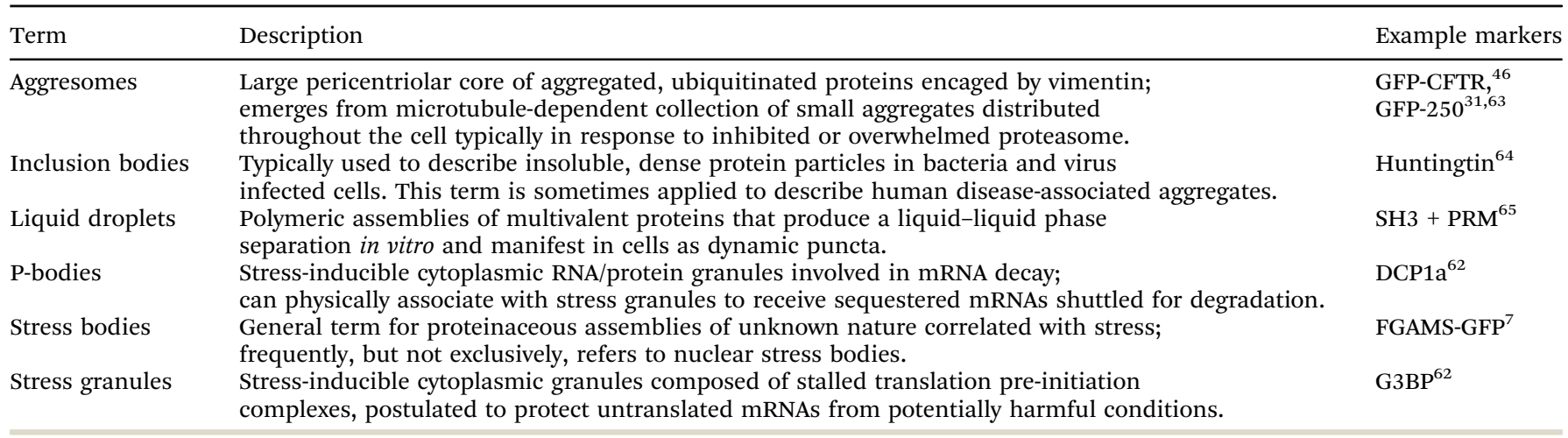

the interpretation of punctate bodies containing purine biosynthetic enzymes (purinosome bodies). Although current observations do not negate the possible formation of functional purinosomes, they also do not suggest that purinosome bodies must be the purinosome. A far simpler explanation is plausible: that the purine biosynthetic enzymes can aggregate under conditions of cellular stress or recombinant expression. Indeed, it is well known that many other intracellular enzymes form aggregates under similar conditions, and purine biosynthetic enzymes are not known to be special in this regard. This discrepancy between purinosome body interpretations highlights the need for caution regarding reliable methodologies for observing punctate body formation and determining their function and physiological relevance.

\section{Acknowledgements}

The authors acknowledge support by grants from the National Institutes of Health, National Science Foundation, Cancer Prevention and Research in Texas, and Welch Foundation to EMM (F-1515) and to ADE (F-1654). MT acknowledges funding and support from Michele Vendruscolo and the University of Cambridge. This review had its inception in discussions of the purinosome on Wikipedia and in PLoS One readers' comments. ${ }^{30}$

\section{References}

1 H. O. Spivey and J. Ovadi, Methods, 1999, 19, 306-321.

2 E. W. Miles, S. Rhee and D. R. Davies, J. Biol. Chem., 1999, 274, 12193-12196.

3 H. M. Holden, J. B. Thoden and F. M. Raushel, Curr. Opin. Struct. Biol., 1998, 8, 679-685.

4 P. Pan, E. Woehl and M. F. Dunn, Trends Biochem. Sci., 1997, 22, 22-27.

5 J. Ovadi, J. Theor. Biol., 1991, 152, 1-22.

6 S. An, R. Kumar, E. D. Sheets and S. J. Benkovic, Science, 2008, 320, 103-106.

7 A. Zhao, M. Tsechansky, J. Swaminathan, L. Cook, A. D. Ellington and E. M. Marcotte, PLoS One, 2013, 8, e56203.

8 F. J. Schendel, Y. S. Cheng, J. D. Otvos, S. Wehrli and J. Stubbe, Biochemistry, 1988, 27, 2614-2623.
9 S. Henikoff, M. A. Keene, J. S. Sloan, J. Bleskan, R. Hards and D. Patterson, Proc. Natl. Acad. Sci. U. S. A., 1986, 83, 720-724. 10 J. Aimi, H. Qiu, J. Williams, H. Zalkin and J. E. Dixon, Nucleic Acids Res., 1990, 18, 6665-6672.

11 E. M. Marcotte, M. Pellegrini, H. L. Ng, D. W. Rice, T. O. Yeates and D. Eisenberg, Science, 1999, 285, 751-753. 12 J. Rudolph and J. Stubbe, Biochemistry, 1995, 34, 2241-2250. 13 E. McCairns, D. Fahey, D. Sauer and P. B. Rowe, J. Biol. Chem., 1983, 258, 1851-1856.

14 P. B. Rowe, E. McCairns, G. Madsen, D. Sauer and H. Elliott, J. Biol. Chem., 1978, 253, 7711-7721.

15 C. A. Caperelli, P. A. Benkovic, G. Chettur and S. J. Benkovic, J. Biol. Chem., 1980, 255, 1885-1890.

16 Y. Deng, J. Gam, J. B. French, H. Zhao, S. An and S. J. Benkovic, J. Biol. Chem., 2012, 287, 36201-36207.

17 L. T. Gooljarsingh, J. Ramcharan, S. Gilroy and S. J. Benkovic, Proc. Natl. Acad. Sci. U. S. A., 2001, 98, 6565-6570.

18 S. An, Y. Deng, J. W. Tomsho, M. Kyoung and S. J. Benkovic, Proc. Natl. Acad. Sci. U. S. A., 2010, 107, 12872-12876.

19 S. An, M. Kyoung, J. J. Allen, K. M. Shokat and S. J. Benkovic, J. Biol. Chem., 2010, 285, 11093-11099.

20 F. Verrier, S. An, A. M. Ferrie, H. Sun, M. Kyoung, H. Deng, Y. Fang and S. J. Benkovic, Nat. Chem. Biol., 2011, 7, 909-915.

21 J. B. French, H. Zhao, S. An, S. Niessen, Y. Deng, B. F. Cravatt and S. J. Benkovic, Proc. Natl. Acad. Sci. U. S. A., 2013, 110, 2528-2533.

22 V. Baresova, V. Skopova, J. Sikora, D. Patterson, J. Sovova, M. Zikanova and S. Kmoch, Hum. Mol. Genet., 2012, 21, 1534-1543.

23 M. S. Field, D. D. Anderson and P. J. Stover, Front. Genet., 2011, 2, 36.

24 N. B. Beaty and M. D. Lane, J. Biol. Chem., 1983, 258, 13051-13055.

25 N. B. Beaty and M. D. Lane, J. Biol. Chem., 1983, 258, 13043-13050.

26 M. J. Meredith and M. D. Lane, J. Biol. Chem., 1978, 253, 3381-3383.

27 B. A. Clarke and S. D. Clarke, Arch. Biochem. Biophys., 1982, 218, 92-100.

28 C. C. Hyde and E. W. Miles, Biotechnology, 1990, 8, 27-32. 29 M. F. Dunn, Arch. Biochem. Biophys., 2012, 519, 154-166. 
30 PLoS One Reader's Comments, Transiently Transfected Purine Biosynthetic Enzymes Form Stress Bodies, http:// www.plosone.org/article/comments/info\%3Adoi\%2F10. 1371\%2Fjournal.pone.0056203.

31 R. Garcia-Mata, Z. Bebok, E. J. Sorscher and E. S. Sztul, J. Cell Biol., 1999, 146, 1239-1254.

32 D. Landgraf, B. Okumus, P. Chien, T. A. Baker and J. Paulsson, Nat. Methods, 2012, 9, 480-482.

33 G. K. Smith, W. T. Mueller, G. F. Wasserman, W. D. Taylor and S. J. Benkovic, Biochemistry, 1980, 19, 4313-4321.

34 G. K. Smith, W. T. Mueller, P. A. Benkovic and S. J. Benkovic, Biochemistry, 1981, 20, 1241-1245.

35 S. A. Broadley and F. U. Hartl, FEBS Lett., 2009, 583, 2647-2653.

36 P. H. Ren, J. E. Lauckner, I. Kachirskaia, J. E. Heuser, R. Melki and R. R. Kopito, Nat. Cell Biol., 2009, 11, 219-225.

37 Y. Chai, S. L. Koppenhafer, N. M. Bonini and H. L. Paulson, J. Neurosci., 1999, 19, 10338-10347.

38 K. Uryu, C. Richter-Landsberg, W. Welch, E. Sun, O. Goldbaum, E. H. Norris, C. T. Pham, I. Yazawa, K. Hilburger, M. Micsenyi, B. I. Giasson, N. M. Bonini, V. M. Lee and J. Q. Trojanowski, Am. J. Pathol., 2006, 168, 947-961.

39 N. R. Jana, M. Tanaka, G. Wang and N. Nukina, Hum. Mol. Genet., 2000, 9, 2009-2018.

40 T. Schmidt, K. S. Lindenberg, A. Krebs, L. Schols, F. Laccone, J. Herms, M. Rechsteiner, O. Riess and G. B. Landwehrmeyer, Ann. Neurol., 2002, 51, 302-310.

41 P. J. McLean, H. Kawamata, S. Shariff, J. Hewett, N. Sharma, K. Ueda, X. O. Breakefield and B. T. Hyman, J. Neurochem., 2002, 83, 846-854.

42 L. V. Kalia, S. K. Kalia, H. Chau, A. M. Lozano, B. T. Hyman and P. J. McLean, PLoS One, 2011, 6, e14695.

43 G. A. Shinder, M. C. Lacourse, S. Minotti and H. D. Durham, J. Biol. Chem., 2001, 276, 12791-12796.

44 P. J. Muchowski and J. L. Wacker, Nat. Rev. Neurosci., 2005, 6, 11-22.

45 M. Bucciantini, E. Giannoni, F. Chiti, F. Baroni, L. Formigli, J. Zurdo, N. Taddei, G. Ramponi, C. M. Dobson and M. Stefani, Nature, 2002, 416, 507-511.

46 J. A. Johnston, C. L. Ward and R. R. Kopito, J. Cell Biol., 1998, 143, 1883-1898.

47 S. Kaminosono, T. Saito, F. Oyama, T. Ohshima, A. Asada, Y. Nagai, N. Nukina and S. Hisanaga, J. Neurosci., 2008, 28, 8747-8755.

48 P. J. Muchowski, K. Ning, C. D'Souza-Schorey and S. Fields, Proc. Natl. Acad. Sci. U. S. A., 2002, 99, 727-732.

49 F. Meggio and L. A. Pinna, FASEB J., 2003, 17, 349-368.

50 M. Watabe and T. Nakaki, J. Cell Sci., 2011, 124, 1519-1532.

51 M. Watabe and T. Nakaki, Commun. Integr. Biol., 2012, 5, 278-280.

52 M. A. Pagano, J. Bain, Z. Kazimierczuk, S. Sarno, M. Ruzzene, G. Di Maira, M. Elliott, A. Orzeszko, G. Cozza, F. Meggio and L. A. Pinna, Biochem. J., 2008, 415, 353-365.
53 C. C. Schneider, A. Hessenauer, C. Gotz and M. Montenarh, Oncol. Rep., 2009, 21, 1593-1597.

54 K. A. Ahmad, G. Wang and K. Ahmed, Mol. Cancer Res., 2006, 4, 331-338.

55 K. A. Ahmad, G. Wang, G. Unger, J. Slaton and K. Ahmed, Adv. Enzyme Regul., 2008, 48, 179-187.

56 J. Tyedmers, A. Mogk and B. Bukau, Nat. Rev. Mol. Cell Biol., 2010, 11, 777-788.

57 A. Williams, L. Jahreiss, S. Sarkar, S. Saiki, F. M. Menzies, B. Ravikumar and D. C. Rubinsztein, Curr. Top. Dev. Biol., 2006, 76, 89-101.

58 E. Ogier-Denis, A. Couvineau, J. J. Maoret, J. J. Houri, C. Bauvy, D. De Stefanis, C. Isidoro, M. Laburthe and P. Codogno, J. Biol. Chem., 1995, 270, 13-16.

59 E. Ogier-Denis, J. J. Houri, C. Bauvy and P. Codogno, J. Biol. Chem., 1996, 271, 28593-28600.

60 M. Kadowaki, R. Venerando, G. Miotto and G. E. Mortimore, J. Biol. Chem., 1994, 269, 3703-3710.

61 P. Anderson and N. Kedersha, J. Cell Biol., 2006, 172, 803-808.

62 N. Kedersha and P. Anderson, Methods Enzymol., 2007, 431, 61-81.

63 R. Garcia-Mata, Y. S. Gao and E. Sztul, Traffic, 2002, 3, 388-396.

64 S. Waelter, A. Boeddrich, R. Lurz, E. Scherzinger, G. Lueder, H. Lehrach and E. E. Wanker, Mol. Biol. Cell, 2001, 12, 1393-1407.

65 P. Li, S. Banjade, H. C. Cheng, S. Kim, B. Chen, L. Guo, M. Llaguno, J. V. Hollingsworth, D. S. King, S. F. Banani, P. S. Russo, Q. X. Jiang, B. T. Nixon and M. K. Rosen, Nature, 2012, 483, 336-340.

66 M. Riedel, O. Goldbaum, L. Schwarz, S. Schmitt and C. Richter-Landsberg, PLoS One, 2010, 5, e8753.

67 C. Mignot, C. Delarasse, S. Escaich, B. Della Gaspera, E. Noe, E. Colucci-Guyon, C. Babinet, M. Pekny, P. Vicart, O. Boespflug-Tanguy, A. Dautigny, D. Rodriguez and D. Pham-Dinh, Exp. Cell Res., 2007, 313, 2766-2779.

68 G. Bjørkøy, T. Lamark, A. Brech, H. Outzen, M. Perander, A. Overvatn, H. Stenmark and T. Johansen, J. Cell Biol., 2005, 171, 603-614.

69 E. Kuusisto, A. Salminen and I. Alafuzoff, Neuropathol. Appl. Neurobiol., 2002, 28, 228-237.

70 E. Kuusisto, A. Salminen and I. Alafuzoff, NeuroReport, 2001, 12, 2085-2090.

71 K. Zatloukal, C. Stumptner, A. Fuchsbichler, H. Heid, M. Schnoelzer, L. Kenner, R. Kleinert, M. Prinz, A. Aguzzi and H. Denk, Am. J. Pathol., 2002, 160, 255-263.

72 U. Nagaoka, K. Kim, N. R. Jana, H. Doi, M. Maruyama, K. Mitsui, F. Oyama and N. Nukina, J. Neurochem., 2004, 91, 57-68.

73 P. J. McLean, H. Kawamata and B. T. Hyman, Neuroscience, 2001, 104, 901-912. 